\title{
Electron-cylotron maser radiation from electron holes: downward current region
}

\author{
R. A. Treumann ${ }^{1,2}$, W. Baumjohann ${ }^{3}$, and R. Pottelette ${ }^{4}$ \\ ${ }^{1}$ Department of Geophysics and Environmental Sciences, Munich University, Munich, Germany \\ ${ }^{2}$ Department of Physics and Astronomy, Dartmouth College, Hanover NH 03755, USA \\ ${ }^{3}$ Space Research Institute, Austrian Academy of Sciences, Graz, Austria \\ ${ }^{4}$ LPP-CNRS/INSU, 94107 Saint-Maur des Fossés, France \\ Correspondence to: R. A. Treumann (rudolf.treumann@geophysik.uni-muenchen.de)
}

Received: 20 October 2011 - Revised: 9 December 2011 - Accepted: 8 January 2012 - Published: 13 January 2012

\begin{abstract}
The electron-cyclotron maser emission theory from electron holes is applied to holes generated in the downward current region of the aurora. It is argued that the main background auroral kilometric radiation source may still be located in the upward current region electron-ring (horseshoe) distribution while the fine structure is caused by electron holes predominantly in the downward current region. There the existence of electron holes is well established and electron densities are high enough for substantial maser growth rates. Trapping of radiation by the holes provides strong amplification. Upward motion of holes favours the escape of radiation both, from the holes and from the downward current region, into the upward current region. Since upward and downward current regions always exist simultaneously, they are acting in tandem in generating auroral kilometric radiation and its fine structure by the same mechanism though in different ways. This mechanism solves the longstanding problem of auroral kilometric radiation fine structure.
\end{abstract}

Keywords. Magnetospheric physics (Auroral phenomena; Plasma waves and instabilities)

\section{Introduction}

Emission of auroral kilometric radiation has commonly been attributed to the upward current region for reasons of observation of electron distributions with sufficiently steep and positive perpendicular velocity space gradients $\partial f_{\mathrm{e}}\left(v_{\|}, v_{\perp}\right) / \partial v_{\perp}>0$ on the electron phase-space distribution $f_{\mathrm{e}}\left(v_{\|}, v_{\perp}\right)$, viz. loss-cone, ring or horseshoe distributions etc. (more precisely the relativistic momentum distribution, cf. e.g. Wu and Lee, 1979; Winglee, 1983; Pritchett, 1984a,b,c,
1986; Louarn, 2006, and others). For a comprehensive review see Treumann (2006). The downward current region has barely been considered a source because none of those distributions is found in that region. The electron component observed here is known to consist purely of ionospheric electrons which, by a sufficiently strong downward field-aligned electrostatic potential of not yet completely clarified origin, are sucked up from the ionosphere and become accelerated into an upward magnetic-field aligned and fairly cold electron beam which carries the downward auroral current (Carlson et al., 1998). Such an electron distribution is believed to be inactive with respect to the electron-cyclotron maser; if it causes radiation, then at the best via a nonlinear wave-wave interaction process like the one known in generating solar type III bursts and emission from the electron foreshock at low harmonics of the electron plasma frequency $\omega_{\mathrm{e}}$. In the auroral region $\omega_{\mathrm{e}} \ll \omega_{\mathrm{ce}}$ is sufficiently below the electron cyclotron frequency $\omega_{\mathrm{ce}}$ for making this mechanism obsolete.

On the other hand, downward field-aligned current strengths are large in the auroral downward current region. It has therefore early been understood that the auroral downward current region would be a preferable location for the generation of phase space holes via some current instability, both ion holes (Berman et al., 1986; Tetreault, 1988, 1991; Gray et al., 1990; Ergun et al., 1998b,c,a) and electron holes (Ergun et al., 2002a,b). Ion holes, in that case, are the result of the ion-acoustic instability evolving from ion acoustic solitons in the presence of dissipation or either from lowerhybrid or from electrostatic whistler waves, in the latter case being of mixed electrostatic/electromagnetic nature. Their dynamics has been investigated in the above papers for parallel propagation. 

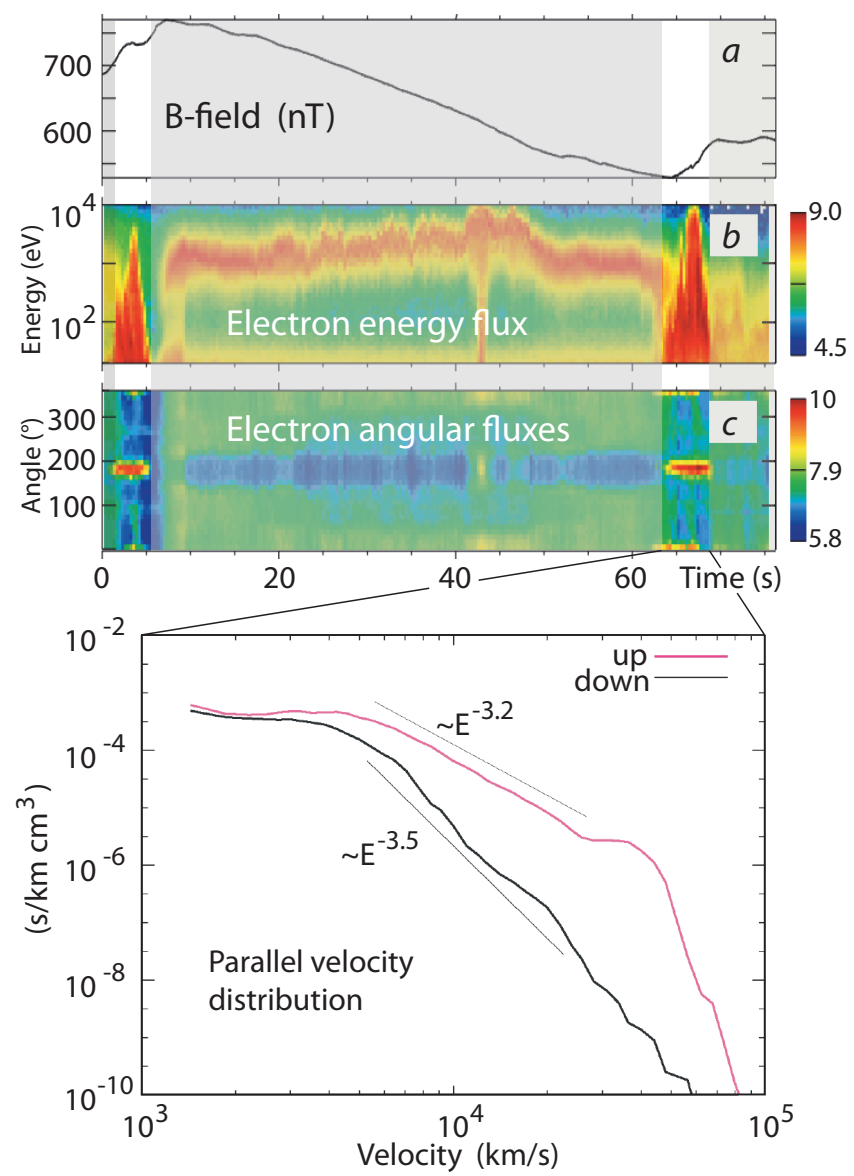

Fig. 1. The two narrow downward current regions of the FAST 1773 orbit flanking the shaded extended upward current region. (a) Magnetic field with the two positive gradient regions indicating downward currents. (b) Electron energy flux. The downward current region is characterised by their typical broad electron energy fluxes. (c) Angular distribution of flux. Showing its concentration at angles of $180^{\circ}$. The parallel electron distribution is shown for the second region in the lower part of the figure (upward electron velocities are negative, here shown mirrored into positive speeds). The low energy flat part is caused by electric-field acceleration. Upward electron fluxes are substantially higher than downward and exhibit a sharp cut-off at high energies after a power law part, indicating strong upward acceleration. The difference between upward and downward fluxes is responsible for the downward current and corresponds to a non-zero upward bulk velocity (Data obtained within the University of California Berkeley - France cooperation).

Like in the upward current region, electron holes would be excited preferably by the Buneman two-stream instability (Buneman, 1958, 1959; Treumann and Baumjohann, 1997). Their dynamics has been the subject of a intense research efforts (Ergun et al., 2002a,b; Muschietti et al., 1999a,b; Newman et al., 2001, 2002; Oppenheim et al., 2001, and others). Neither of these structures have been considered to be of any importance in the generation of radiation, however. Their mentioning in connection to radiation was entirely restricted to the upward current region and to a few papers only (Pot- telette et al., 2001; Pottelette and Treumann, 2005; Pottelette and Pickett, 2007; Treumann, 2006; Treumann et al., 2008). In a recent paper (Treumann et al., 2011a) we developed the theory of electron-hole maser radiation in the upward current region.

In the present communication we transform the latter theory to the downward current region. This attempt is stimulated by the discouraging result obtained in the previous paper when applying the electron-hole maser to the upward current region. There we combined high-resolution observations with simulations of electron holes and radiation theory. We found that electron holes can indeed be emitters of narrowband $\mathrm{X}$-mode radiation via the electron-cyclotron maser. The emitted bandwidths and emission frequencies fall all into place when compared with observation. Also, the polarisation of the radiation turned out to be correct, on the X-mode (or Z-mode) branch. However, we were discouraged by the low growth and amplification rates of the radiation.

In an attempt to deal with this problem, we realised that the radiation was sufficiently short wavelength and low frequency for being completely trapped inside the electron hole. When the radiation is generated at the electron hole boundary though outside the hole, then the holes do indeed not play any role in radiation production because the radiation does not stay long enough in contact with the hole for reaching large enough amplitudes. Trapping is, however, suggested when the radiation is generated inside the hole on its inner boundary by the fact that the hole is low density and the radiation cannot escape but is confined for the entire lifetime of the hole. For this time it stays in resonance and is continuously amplified. Nevertheless, in the upward current region the amplification factor is small because of the very low electron density and the resulting very small growth rates. In addition, after decay of the hole and release of the radiation its frequency is still far below the X-mode cut-off, and the radiation can propagate only in the Z-mode. Though Z-mode radiation has indeed been observed in the auroral kilometric source region, its escape to free space poses another problem (cf. e.g. Louarn, 2006, and references therein).

The downward current region has the advantage of hosting much higher electron densities than in the auroral cavity. Still, these densities are low enough for igniting the electron cyclotron maser instability if only the electron distribution exhibits a sufficiently steep perpendicular velocity gradient. This, however, can be generated locally by electron holes. Any radiation excited by the electron-cyclotron maser inside the holes, being trapped for the lifetime of the hole and afterwards released, can then leak out from the downward current region into the upward current region where it may contribute to fine structure and, ultimately, may escape to free space from the macro-scale auroral cavity. Here we show that electron holes in the downward current region are indeed capable of this. Their growth and amplification rates are substantially higher than in the upward current region, and their frequencies are above the upward current region X-mode cut-off. 

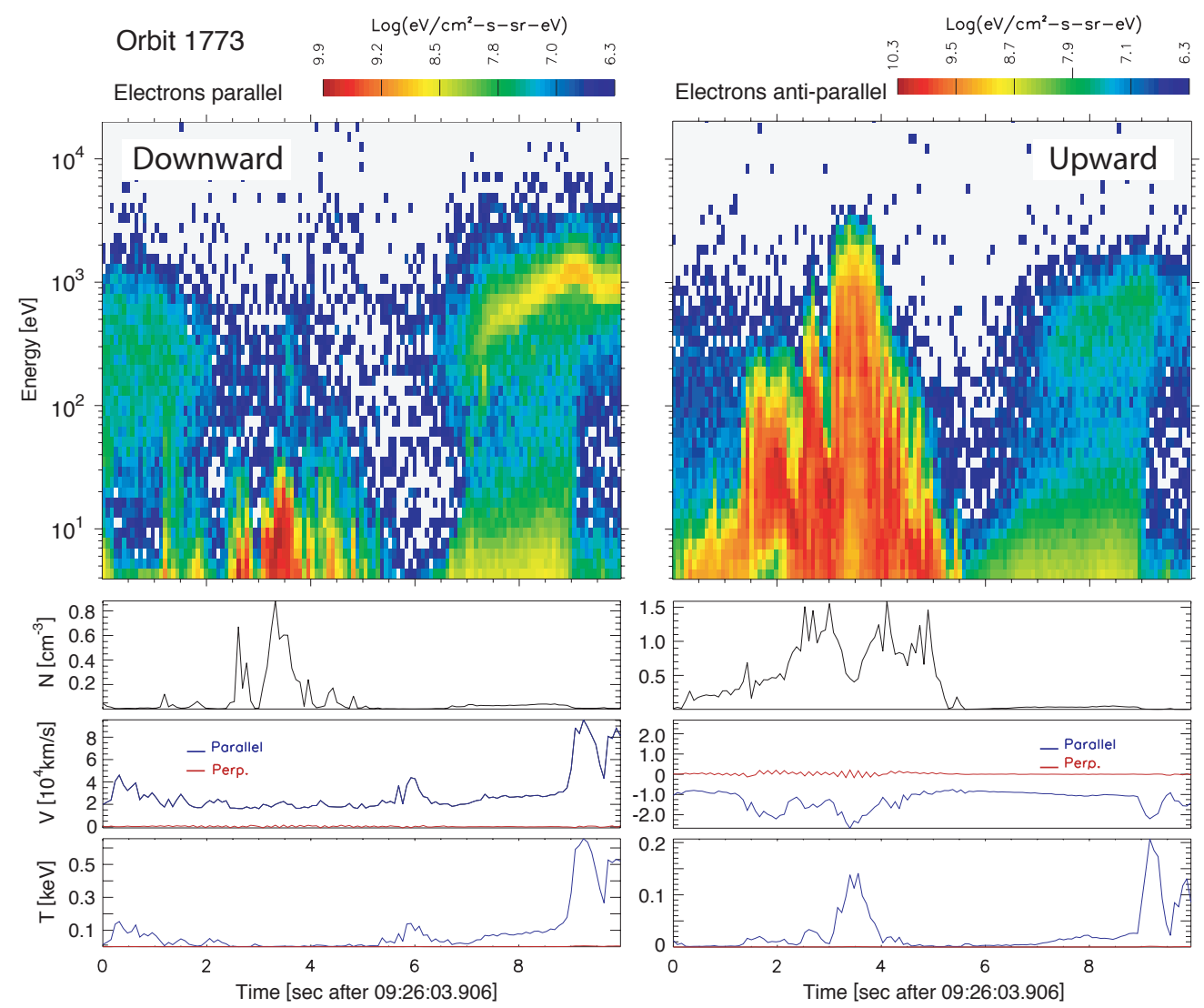

Fig. 2. A high resolution view of the second downward current event in Fig. 1 for downward (left) and upward (right) electron fluxes. Downward fluxes [i.e. the $v_{\|}>0$ tail of $f_{\mathrm{e}}\left(v_{\|}\right)$] are low energy $\sim 10 \mathrm{eV}$ with density $\lesssim 1 \mathrm{~cm}^{-3}$. [Downward bulk velocities between $2 \mathrm{~s}<$ $t<4 \mathrm{~s}$ are formally obtained from the downward part of $f_{\mathrm{e}}\left(v_{\|}\right)$, i.e. the downward tail of the distribution function, and are not real.] Upward fluxes reach densities of $\gtrsim 1.5 \mathrm{~cm}^{-3}$. Plasma frequencies in this region range around $10 \mathrm{kHz} \lesssim \omega_{\mathrm{e}} / 2 \pi \lesssim 14 \mathrm{kHz}$. Upward bulk velocities range between $10^{4} \mathrm{~km} \mathrm{~s}^{-1} \lesssim V<2.5 \times 10^{4} \mathrm{~km} \mathrm{~s}^{-1}$ while temperatures maximise at $T \sim 150 \mathrm{eV}$. Thus the thermal velocities are $v_{\mathrm{e}}<5 \times 10^{3} \mathrm{~km} \mathrm{~s}^{-1}$. Under these conditions the Buneman instability grows resulting in formation of chains of electron holes.

\section{Relevant downward current region observations}

Panels (a)-(c) of Fig. 1 present the trace of the magnetic field, electron energy and angular fluxes, respectively, for the FAST 1773 orbit. Observations in the broad upward current region (shaded here) have been described exhaustively in Treumann et al. (2011a). It is flanked by two downward current regions, as indicated by the positive field gradients in panel (a), for the first $10 \mathrm{~s}$ and $65-80 \mathrm{~s}$. The second of these will be analysed in more detail below. Electron fluxes maximise at few $10 \mathrm{eV}(\sim 100 \mathrm{eV})$ and extend up to few $\mathrm{keV}$ $(\sim 10 \mathrm{keV})$. Angular distributions peak strongly at $\sim 180^{\circ}$ antiparallel to the local magnetic field direction (angular resolution is $\pm 11^{\circ}$ ) with weak fluxes at oblique angles. The ions have energies of few $\mathrm{keV}$, being distributed into conics (very low upward ion fluxes).

In the lower part of Fig. 1 the time-averaged electron velocity distribution function along the magnetic field is shown, separated into upward and downward distributions for the second downward current event. The distributions exhibit the excess of upgoing electrons at velocities above $v \sim 2 \times 10^{3} \mathrm{~km} \mathrm{~s}^{-1}$ peaking at $v \sim 4 \times 10^{3} \mathrm{~km} \mathrm{~s}^{-1}$ before turning into a power law decay for roughly one decade in velocity until $v \sim 3 \times 10^{4} \mathrm{~km} \mathrm{~s}^{-1}$. This power law decay is indicated as the corresponding power law $\sim \mathcal{E}^{-3.2}$ in the equivalent parallel energy distribution. It is followed by an exponential cut-off at velocities $v>4 \times 10^{4} \mathrm{~km} \mathrm{~s}^{-1}$. [Note that the flat top at low speeds is typical for distributions accelerated by stationary electrostatic fields (for the latter see Andersson et al., 2002).] Just before the cut-off, the upward bump on the distribution indicates the presence of a change in the average character of the distribution which resembles a beam bounding the distribution function.

We plot in Fig. 2 the electron data and moments of the distribution for this second downward current region in higher resolution. On the left are the downward electron fluxes, on the right the current-carrying upward fluxes. Only these are of interest when anticipating that the downward fluxes on the left are residual downward directed Maxwellian tails of the electron distribution, possibly mixed with a small amount of 


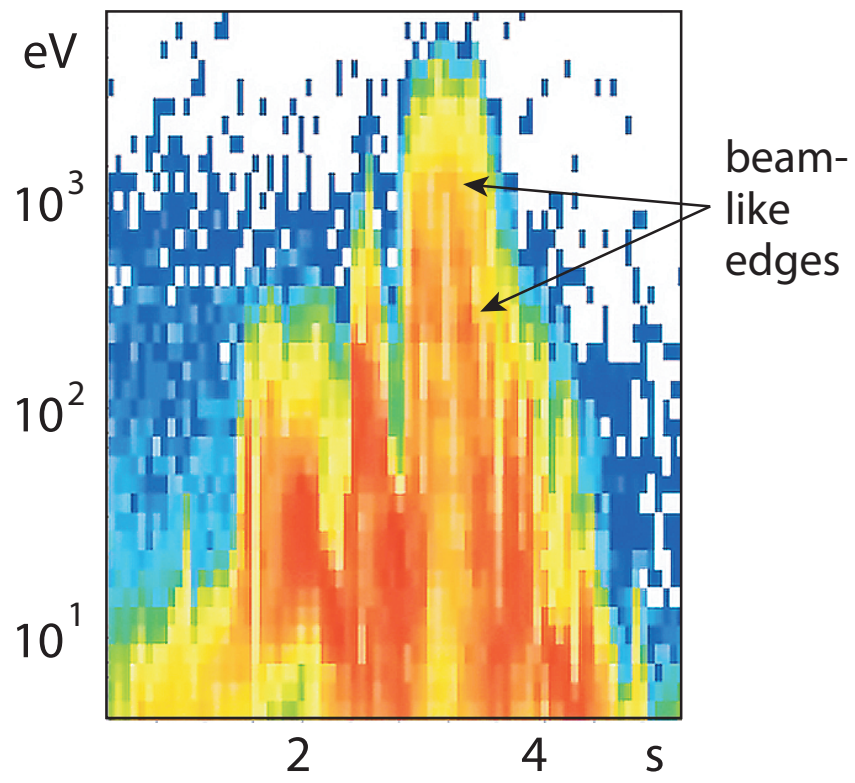

Fig. 3. Expanded view of the second downward current event in Fig. 2, showing that the upward electron fluxes consists of several neighbouring events each one being sharp-edged in energy with intensified energy flux close to the highest energies at the particular times. These edge features add up to the average high-energy beamlike increase in the upward electron distribution function of Fig. 1.

nonlinearly - electron-hole backscattered - downward accelerated electrons. All upward electron fluxes exhibit a very high temporal dynamic, suggesting that the upward currentcarrying electron fluxes originate from many closely spaced narrow acceleration regions of temporal extension $<1 \mathrm{~s}$ with overlapping electron fluxes.

There are several noticeable observations which we briefly list as follows:

- First, the total electron density of upward and downward electrons amounts to roughly $N \approx 2 \mathrm{~cm}^{-3}$ which corresponds to a plasma frequency of $\omega_{\mathrm{e}} / 2 \pi \sim 13 \mathrm{kHz}$. This value is still far below the local electron cyclotron frequency and emission frequency of auroral kilometric radiation which in this case was $\omega_{\mathrm{ce}} / 2 \pi \sim 483 \mathrm{kHz}$ (Treumann et al., 2011a). Thus the ratio $\omega_{\mathrm{e}} / \omega_{\mathrm{ce}} \sim$ $3 \times 10^{-2} \ll 1$ indicates that the electron cyclotron maser radiation theory from electron holes will in principle be applicable in its ordinary version but will lead to larger hole-maser-growth rates than were obtained in the upward current region.

- Second, the upward velocity of the current-carrying electrons reaches values $V>2 \times 10^{4} \mathrm{~km} \mathrm{~s}^{-1}$ with an average value of $\langle V\rangle \sim 1.8 \times 10^{4} \mathrm{~km} \mathrm{~s}^{-1}$, roughly a factor of $\gtrsim 2$ larger than the electron thermal velocity $v_{\mathrm{e}}<$ $10^{4} \mathrm{~km} \mathrm{~s}^{-1}$. This is sufficiently high for the Buneman instability to be excited. Thus the conditions for generation of electron holes are satisfied in the downward current region. Ion velocities (not shown) are downward being of the order of few times $100 \mathrm{~km} \mathrm{~s}^{-1}$. This velocity adds to the downward current velocity thereby improving the above condition. Moreover, the existence of phase space holes, ion holes as well as electron holes, both on the Debye scale, has been confirmed experimentally here (Carlson et al., 1998; Ergun et al., 1998a,b,c).

- Third, inspection of the electron spectrum (see the enlarged version in Fig. 3) indicates that at any given time the electron fluxes near their high-energy ends - in the figure indicated by arrows - generally terminate with increases before being cut-off exponentially. These have added up to form the beam-like feature on the averaged upward electron distribution in the bottom part of Fig. 1, where the large spread in velocity is caused by averaging over the highly variable fluxes thus faking a much higher beam temperature and lower beam phase space density. The instantaneous about realistic width in energy - or temperature - of this feature can be read from the maximum in energy at second 3.5 in Fig. 3 to amount to $\sim 100 \mathrm{eV}$ (at the 1 pixel energy resolution) yielding a thermal speed of $v_{\mathrm{eb}} \sim 10^{3} \mathrm{~km} \mathrm{~s}^{-1}$ for this feature. It is suggestive to take it as indication of the cold electron beam seen in the numerical simulations (Newman et al., 2001) of electron holes, the nonlinearly "electron-hole cooled" residuum of the initial warm electron-current "beam" distribution (cf. Fig. 7 in Treumann et al., 2011a).

\section{Downward current region electron holes}

The mechanism of generation of electron holes in the downward current region is the same as that in the upward current region. The only differences are:

- the absence of a ring-horseshoe electron distribution which is replaced by the upward electron flow distribution in Fig. 1,

- the substantially higher electron plasma frequency,

- the propagation direction of the electron holes, which by theory is in the same direction as the electron "beamcurrent" velocity (caused by the Buneman instability the hole speed $V_{\mathrm{h}}<V$ is slightly below the current speed; thus, in the electron beam frame, the holes move against the beam); in the downward current region it should be upward towards lower electron cyclotron frequencies, and

- the question of the perpendicular extension of the electron holes in velocity space. 
Like in the upward current region, the extension of holes in real (configuration) space is of little or secondary importance. It comes into play when considering the possible trapping and amplification of radiation in Sect. 4. What is absolutely necessary (cf. e.g. Treumann, 2006) for the electroncyclotron maser to work, is a perpendicular velocity gradient on the electron phase space distribution function and the presence of mildly relativistic electrons in resonance (known since the seminal work of Wu and Lee, 1979). Again, this latter point is crucial. Ample arguments for a perpendicular hole structure in velocity space were provided for holes in the upward current region. These will have to be repeated and modified below.

Our simplifying assumption is that the electron holes will be excited by the Buneman two-stream instability (Buneman, 1958, 1959; Treumann and Baumjohann, 1997). This requires that, for a given fixed perpendicular velocity the effective electron drift along the magnetic field $v_{\|}\left(v_{\perp}\right)>v_{\mathrm{e}}\left(v_{\perp}\right)$ should exceed the effective parallel electron thermal velocity at this particular fixed perpendicular speed in order to excite the Buneman mode at this fixed $v_{\perp}$. The angular fluxes in Fig. 1 suggest that there is only a small spread of the distribution into perpendicular direction. However, time, angle and energy resolutions do not permit for any precise conclusion on the scale of the electron holes, neither in configuration nor in velocity space.

From theory and simulations it is known that the spatial extension of the holes along the magnetic field is of the order of $\lesssim 100 \lambda_{\mathrm{D}}$, with Debye length $\lambda_{\mathrm{D}} \lesssim 50 \mathrm{~m}$ at $T_{\mathrm{e}} \sim 100 \mathrm{eV}$ and $N \approx 2 \mathrm{~cm}^{-3}$ yielding a parallel scale of few $\mathrm{km}$. The thermal gyroradius of electrons in this case is $r_{\mathrm{ce}}^{\text {th }} \sim 10 \mathrm{~m}$ (one should note that in Fig. 2 the perpendicular temperature given in red is unreliable, for the measured particle numbers were rather to small). A $1 \mathrm{keV}$ electron (as in Fig. 2) under these conditions has gyroradius $r_{\mathrm{ce}} \sim 30 \mathrm{~m}$. Hence gyroscale-limited electron holes should be rather elongated along the magnetic field. Simulations in weak fields show instead (Newman et al., 2002; Oppenheim et al., 2001) oblate holes. Observations in the strong auroral magnetic field region (Franz et al., 2000) suggest, on the other hand, that they are about spherical in configuration space, though no distinction is made between the upward and downward current regions. This larger spatial extension at small electron gyroradii is reasonable if more than one electron gyro-fluxtube is involved into a hole. However, the electron velocity distribution is independent of the spatial form of the hole, unless the electrons would be excessively heated into perpendicular direction.

Little is known about the exact perpendicular form of the hole and the range of perpendicular velocities affected by the hole. We may try to use the observations in Fig. 1 to infer about the range of observed perpendicular velocities. The data at $t \lesssim 6 \mathrm{~s}$ suggest that the angular distribution spreads out between $\sim 150^{\circ}$ and $\sim 230^{\circ}$. Perpendicular flux levels are very uncertain here. At the higher fluxes

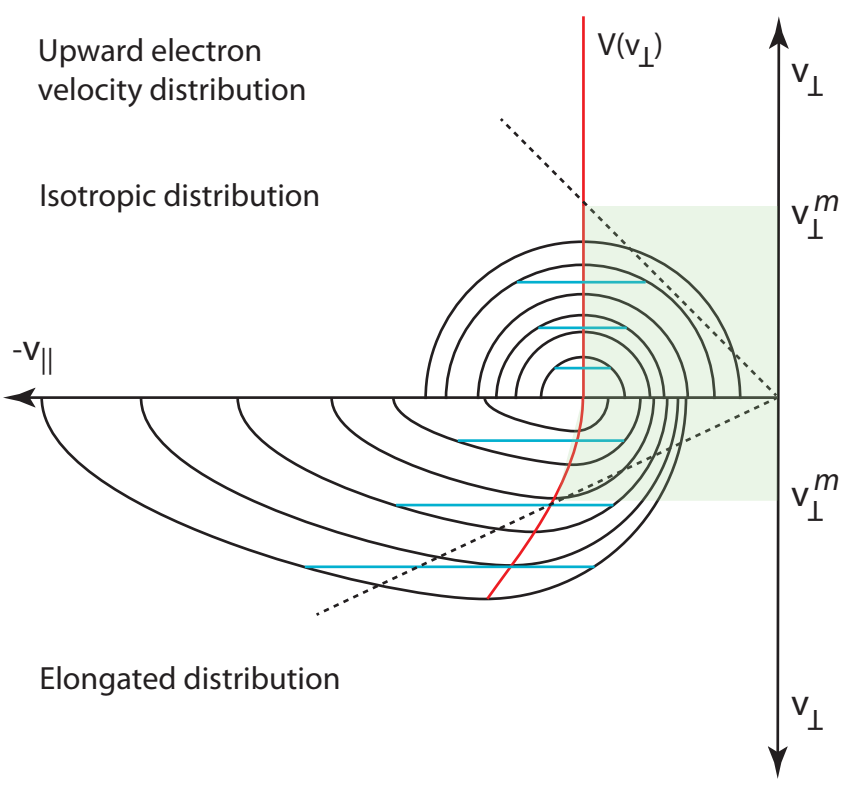

Fig. 4. Sketch of the upward electron distribution in velocity space. Upper half plane: isotropic shifted distribution. The shift is the (negative) upward electron velocity $V$ (shown in red). The (blue) horizontals indicate the "thermal spread" $v_{\mathrm{e}}\left(v_{\perp}\right)$ at three fixed perpendicular velocities $v_{\perp}$. Lower half plane: same for an elongated electron distribution (as implied by the measured parallel electron distribution in Fig. 1). In this case both current speed $V\left(v_{\perp}\right)$ and "thermal spread" $v_{\mathrm{e}}\left(v_{\perp}\right)$ increase at a larger amount with increasing $v_{\perp}$ than in the isotropic case. Dashed lines indicate the maximum Buneman-unstable angles $\theta$ in velocity space. Green-shaded domains indicate ranges of Buneman-unstable perpendicular velocities $v_{\perp}$ smaller than the maximum nominally unstable $v_{\perp}^{m}$. This domain is reduced for the elongated distribution suggesting that for the real elongated case the hole assumes elliptic shape in velocity space. Maser emission is then intrinsically oblique.

in the time interval $65 \mathrm{~s} \lesssim t \lesssim 75 \mathrm{~s}$ the flux spreads over the entire angular range from parallel to perpendicular, though at low about constant flux (green on the colour bar being roughly 1.6 to 2.2 orders below maximum flux). We can use this observed flux ratio $F_{\|} / F_{\perp}$ for a very rough estimate of the possible range of perpendicular velocities. Since fluxes are $F \propto v^{3}$ - densities are not affected - we have $10^{-2.2}<\left(v_{\perp} /\langle V\rangle\right)^{3}<10^{-1.6}$ which yields $3.7 \times 10^{3}<v_{\perp} \lesssim$ $6 \times 10^{3} \mathrm{~km} \mathrm{~s}^{-1}$. Compared to the nominal isotropic thermal speed of $v_{\mathrm{e}}<4.5 \times 10^{3} \mathrm{~km} \mathrm{~s}^{-1}$ (temperature $T_{\mathrm{e}} \lesssim 150 \mathrm{eV}$ ), the larger of these values of $v_{\perp}$ is marginally high for the Buneman instability to be excited.

There is, however, little reason to assume that the distribution would indeed be isotropic with perpendicular temperature equalling the parallel temperature. Upward electrons are accelerated out of the upper ionospheric background distribution which has temperature $<10 \mathrm{eV}$ corresponding to thermal velocities $v_{\mathrm{e}}<1.2 \times 10^{3} \mathrm{~km} \mathrm{~s}^{-1}$. In an accelerating upward electric potential drop and collisionless conditions the 


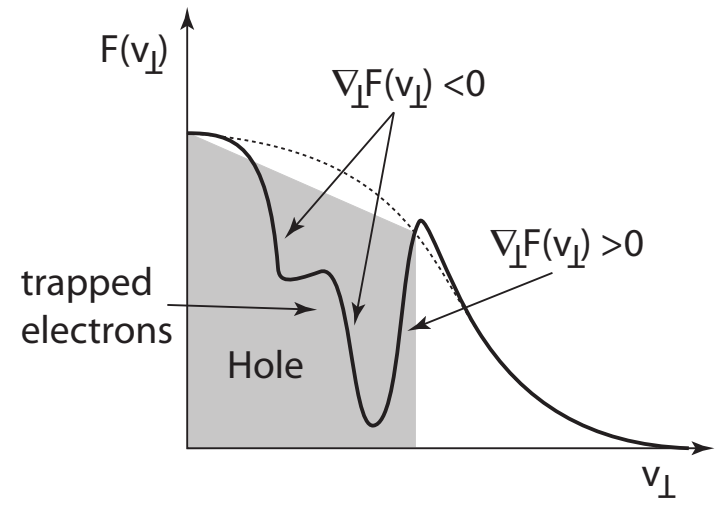

Fig. 5. A model electron distribution in the hole. The hole cuts out part of the distribution but also traps electrons. This causes a positive velocity space gradient at the high velocity space boundary of the hole and a negative gradient at the trapped electron distribution. Radiation is generated at the high electron velocity gradient and absorbed at the low velocity gradient though at different frequencies.

electrons should barely become accelerated into perpendicular direction while parallel heating is a well-known fact being partially due to the action of the holes. At the contrary, for the perpendicular velocity, the magnetic mirror force should even cool them perpendicularly when moving up the field line, the effect contrary to the well know perpendicular heating in the upward current region. Hence, perpendicular temperatures (red in Fig. 1 where they were not measurable) will probably be lower being closer to electron temperatures in the upper ionosphere.

We therefore assume that, independent of the shape of the electron hole in configuration space and independent of the strength of the magnetic field - which determines the gyroradius of the electron and thus the perpendicular spatial extension of the hole -, the perpendicular electron velocity probably spans a sufficient range allowing the hole in velocity space to extend to perpendicular speeds up to which the Buneman instability can be excited at finite $v_{\perp}$. The simple condition to be satisfied at any given $v_{\perp}$ is that, for the Buneman two-stream instability to be excited, the median parallelcurrent speed

$V\left(v_{\perp}\right)>v_{\mathrm{e}}\left(v_{\perp}\right)$

exceeds the thermal velocity $v_{\mathrm{e}}\left(v_{\perp}\right)$ at this particular $v_{\perp}$.

To find out up to which maximum electron pitch angle $\theta$ (or perpendicular speed) this will happen, we return - for simplicity - to the unfavourable isotropic temperature distribution yielding a Maxwellian distribution shifted in upward velocity at the amount of the downward current speed $\langle V\rangle$ and parallel thermal speed $v_{\mathrm{e}}$. This might not be entirely correct even in parallel direction as the lower part of Fig. 1 suggests that the parallel electron distribution is elongated to high parallel velocities, which introduces some correction to the symmetric assumption but is secondary for the main purpose of this investigation.

Since $V \equiv\langle V\rangle=$ const in the symmetric case is independent of $v_{\perp}$. the "effective parallel thermal velocity" is easy to determine. The result (Fig. 4) is that the perpendicular velocity for which the Buneman condition is satisfied at a given fixed $v_{\perp}$ is limited by

$v_{\perp}<v_{\perp}^{\max } \equiv\langle V\rangle\left(1-\frac{v_{\mathrm{e}}^{2}}{\langle V\rangle^{2}}\right)^{\frac{1}{2}}$

or electron pitch angle

$\theta<\theta_{\max }=\sin ^{-1}\left(1-\frac{v_{\mathrm{e}}^{2}}{\langle V\rangle^{2}}\right)^{\frac{1}{2}}$

Inserting $\langle V\rangle=1.8 \times 10^{4} \mathrm{~km} \mathrm{~s}^{-1}$, and $v_{\mathrm{e}} \sim 5 \times 10^{3} \mathrm{~km} \mathrm{~s}^{-1}$ we obtain for the isotropic distribution $\theta \lesssim 70^{\circ}$.

These conditions are simpler than in the case of the ring-horseshoe distribution of the upward current region (Treumann et al., 2011a) because, using the isotropic distribution, it is implicitly assumed that the current velocity $V\left(v_{\perp}\right)$ as function of $v_{\perp}$ does not change with angle to the magnetic field. The more realistic elongated distribution case becomes more stringent, because the "effective parallel thermal speed" increases with increasing $v_{\perp}$, as is obvious from the sketch in the lower part of Fig. 4. In this case also the current speed $V\left(v_{\perp}\right)$ varies with $v_{\perp}$. This, however, is of little importance for our general assumption that a limited range of perpendicular velocities exists in velocity space for which the distribution at given $v_{\perp}$ will become Buneman unstable and electron holes can be generated at finite perpendicular velocity.

Within the limiting electron pitch angle the electron hole extends into perpendicular velocities in phase space. Since the hole is characterised by a depletion of electrons inside the hole and a snowplough effect on the surrounding distribution in the course of which a fast, comparably dense and cold electron beam is created, the hole self-consistently produces a velocity gradient on the distribution function at its phase space boundary not only in the parallel velocities but also in perpendicular velocities.

\section{Radiation}

With these explicit preliminaries in mind we can straightforwardly apply the electron-cyclotron maser theory of electron holes developed in our previous communication (Treumann et al., 2011a). A perpendicular model hole electron velocity distribution is shown in Fig. 5.

\subsection{Growth rate}

Instead of the ring distribution we have in this case the isotropic dense upstreaming electron distribution which at 
electron velocities outside the hole is bounded by accelerated electron beams of density $N_{\mathrm{eb}}$. Working in the hole frame and applying to the circular hole case the resonance condition becomes a circle of radius

$R_{\mathrm{res}}=\sqrt{2\left(1-v_{\mathrm{ce}}\right)}$

with $v_{\mathrm{ce}}=\omega / \omega_{\mathrm{ce}}$ and $\omega_{\mathrm{ce}}=e B / m_{\mathrm{e}}$ the non-relativistic electron cyclotron frequency. Like for upward-current region holes the wave propagates in the X-mode, strictly perpendicular to the magnetic field in this simplified case of a circular hole. The theory at this point is exactly the same as that for radiation from holes in the upward current region as formulated in the hole frame (cf. Sects. 5.2-5.6 of Treumann et al., 2011a). Formulation in the hole frame assures strictly perpendicular radiation up to minor corrections due to a possible non-circular shape of the hole, which we neglect. Since the hole, being the source of radiation, moves at large upward velocity $V_{\mathrm{h}} \lesssim\langle V\rangle$, the final result must again be relativistically transformed back into the stationary observers frame. Even this step is the same as in the upward current region where it was investigated in detail, causing Doppler shift in frequency and obliquity of radiation.

Calculation of growth rates exactly follows Eqs. (8)-(9) and Eqs. (15)-(17) of our former paper (Treumann et al., 2011a) with the only difference that in the downstream current region the integration path around the hole can be taken over the full angular interval $0 \leq \phi \leq \pi$, because this time the hole is entirely embedded into the upward electron distribution. This increases the growth rate by a factor $\lesssim 2$.

The maximum growth rate is obtained at maximum resonance

$R_{\mathrm{res}}^{m} \approx U-\frac{(\Delta u)^{2}}{U}$

where $U=V / c, \Delta u=v_{\mathrm{e}} / c$, with $V \sim\langle V\rangle$ and $v_{\mathrm{e}}$ the thermal speed. This yields a maximum normalised growth rate

$\operatorname{Im}\left(v_{\mathrm{ce}}\right) \approx\left(\frac{\alpha \pi^{3}}{4}\right)\left(\frac{\omega_{\mathrm{e}}^{2}}{\omega_{\mathrm{ce}}^{2}}\right)\left(\frac{\omega_{\mathrm{ce}}}{\omega}\right)$

The plasma frequency is based on the total electron density $N$, while $\alpha=N_{\mathrm{eb}} / N \sim 10^{-2}$ is the ratio of the fast accelerated electron beam to background densities, taken as the electron flux ratio of the bump at $v=5 \times 10^{4} \mathrm{~km} \mathrm{~s}^{-1}$ to the plateau in the distribution function at the bottom in Fig. 1. The precise value of $\alpha$ is not known; the above number is a time average taken over the entire highly variable event (see Fig. 3). It might be larger for single holes up to, say, one order of magnitude at most. Thus $\alpha \sim 1 \%$ is probably a conservative choice. With measured frequencies $\omega_{\mathrm{e}} / 2 \pi \approx 15 \mathrm{kHz}$, $\omega_{\mathrm{ce}} / 2 \pi \approx 480 \mathrm{kHz}$ we have

$\operatorname{Im}(\omega) \equiv \omega_{\mathrm{ce}} \operatorname{Im}\left(v_{\mathrm{ce}}\right) \gtrsim 2 \times 10^{-4}\left(\frac{\omega_{\mathrm{ce}}}{\omega}\right) \frac{\omega_{\mathrm{ce}}}{2 \pi}$
The emission frequency $\omega \lesssim \omega_{\text {ce }}$ is very close to the electron cyclotron frequency such that it does not contribute substantially to the growth rate. Due to the substantially enhanced density in the downstream current region, this value is roughly two orders of magnitude higher than the corresponding value of the electron hole-maser growth rate obtained in the upward current region. For the measured values the growth rate becomes

$\operatorname{Im}(\omega) \approx 10^{2} \mathrm{~Hz}$

Electron-cyclotron maser emission from electron holes in the downward current region is thus much stronger than from the upward current region. Still, the linear growth rate is small and thus the radiation weak unless it becomes further amplified; since, however, in the downward current region no other source of the electron cyclotron maser exists, any electron cyclotron-maser radiation originating in this region is probably exclusively due to the presence of electron holes in the downward current region.

What concerns the radiation bandwidth $\Delta \omega$ at resonance, we may again refer to the arguments given in the case of the upstream current region. Like in that case the hole moves at speed $V_{\mathrm{h}}$ with respect to the stationary observer's frame. Hence, seen from the stationary frame, the resonances which contribute to radiation, i.e. the radii $R$ of resonance circles cutting through the narrow boundary of the hole, are limited to the range $R_{\min }<R<R_{\max }$ which corresponds to a resonant frequency range

$\Delta v_{\mathrm{ce}} \equiv v_{\max }-v_{\min }=\frac{1}{2}\left(R_{\max }^{2}-R_{\min }^{2}\right)$

Since the hole is small, this range is determined by the extension of the hole in parallel velocity $\Delta u_{\|} \approx$ few $\beta_{\mathrm{e}}$, as we have shown (Treumann et al., 2011a), where $\beta_{\mathrm{e}}=v_{\mathrm{e}} / c$. Hence, with observed parallel electron temperatures of the order of $T_{\mathrm{e}} \lesssim 150 \mathrm{eV}$ it becomes $\Delta v_{\mathrm{ce}} \sim 1 \%$, which at emission frequency $\omega / 2 \pi \approx 480 \mathrm{kHz}$, as in our case, is in frequency about $\Delta \omega / 2 \pi<5 \mathrm{kHz}$, clearly a narrow-band fine structure in the auroral kilometric radiation and in agreement with observed bandwidths of fine structure.

\subsection{Amplification}

Electron-cyclotron maser radiation from electron holes is weak not only in the upward but also in the downward current region unless it undergoes further amplification. Only then can it explain any of the observed fine structures in the auroral kilometric radiation (or maser radiation from other planets, the sun and other astrophysical objects, cf. e.g. Zarka and Kurth, 2005; Hess et al., 2009; Louarn et al., 2007; Mottez et al., 2010; Treumann et al., 2011b; Bykov and Treumann, 2011, and references therein).

Radiation that would be generated outside the electron hole in the perpendicular velocity gradient between the hole and the fast accelerated beam or between the beam and the background distribution will not experience amplification. 


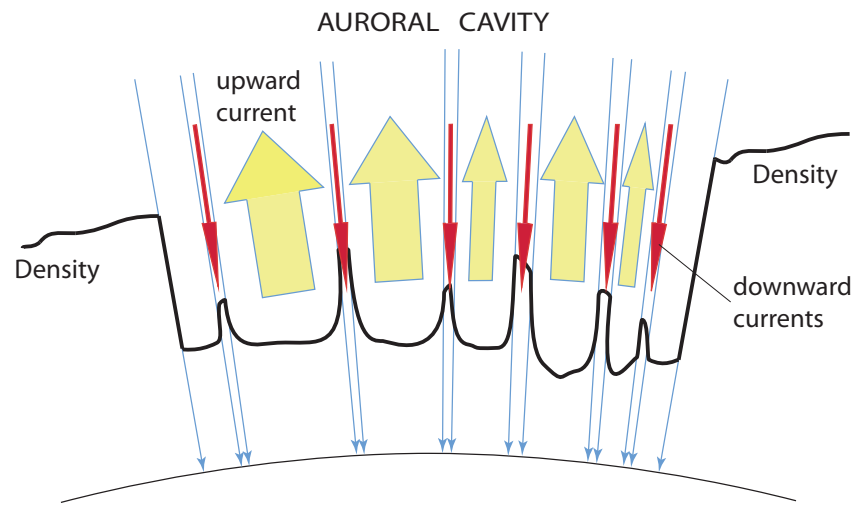

Fig. 6. Sketch of auroral cavity which is bounded by magnetic field lines (blue) and contains a large number of broad upward current regions (yellow upward arrows) and narrow downward current regions (red downward arrows) the latter having higher density than the former but less than outside the auroral cavity. Any radiation emitted in either of these regions will be trapped in the auroral cavity bouncing back and forth between its boundaries until escaping upward into free space. It should be clear from the figure that any radiation that has escaped from its small-scale source (e.g. electron hole as well as local distribution) will barely encounter any other favourable resonance at same frequency in the auroral cavity.

Radiation of this kind is below the X-mode cut-off, propagating in the $\mathrm{Z}$ mode, and is thus confined to the plasma but is free with respect to the hole and escapes from its location. It gets readily out of resonance with the radiation source, barely encountering anymore any favourable perpendicular velocity gradient for being further amplified. It thus may contribute to a weak radiation background filling the auroral cavity and not discriminating between the upward and downward current regions.

In order to experience substantial amplification, the electron-cyclotron maser radiation produced by phase space holes must necessarily be trapped inside the hole for staying long enough in resonance with the steep perpendicular velocity space gradient at the hole boundary for achieving large amplification rates. Such radiation is generated inside the hole and, because of its frequency being below the $\mathrm{X}$ mode cut-off in the hole, is trapped for the entire lifetime of the hole. By maser theory, its frequency is below the local electron cyclotron frequency. Therefore, it cannot escape to the hole environment because of the even higher external $\mathrm{X}$-mode cut-off frequency outside the hole. It bounces back and forth inside the hole between the hole boundaries. Trapping of radiation we have also proposed for radiation from electron holes in the upward current region (Treumann et al., 2011a).

Electron hole life times have been suggested to be limited by the transverse instability of holes (Oppenheim et al., 2001; Newman et al., 2002; Ergun et al., 2002a,b). Simulations fixed the life times of holes against transverse insta-
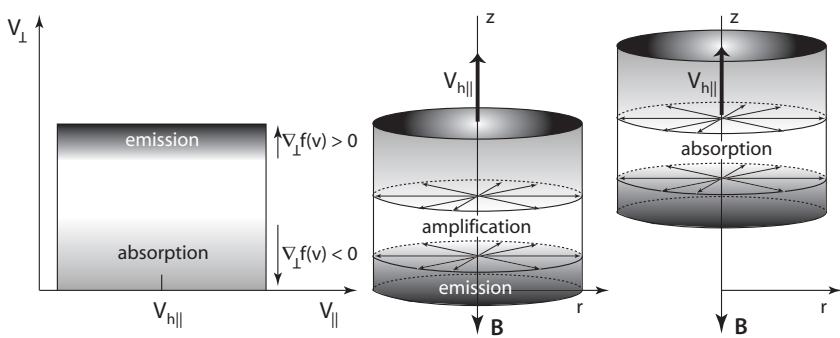

Fig. 7. Electron-cyclotron maser radiation generated and trapped in an idealised cylindrical hole moving with speed $v_{\mathrm{h} \|}$ along the magnetic field from strong to weak magnetic field strengths $B$. Left: the hole in velocity space, $v_{\|}, v_{\perp}$, showing the regions of velocity space gradients where emission and absorption is expected to take place. Right: real space trapping of radiation inside the hole. Radiation trapping (indicated by two-sided arrows in radial perpendicular direction, i.e. the direction of wave propagation) takes place in radial direction, $r$, at any azimuthal angle perpendicular to $\mathbf{B}$. The trapped wave is emitted at resonant frequency $\omega$ with narrow bandwidth $\Delta \omega$. Shown is radiation emitted at the high-frequency end of the hole in the strong magnetic field where the radiation is in resonance and is amplified. When the hole moves up to lower fields (shown on right), radiation at given frequency $\omega$ enters the absorption band and experiences damping. Radiation will be released in X mode at residual intensity when the hole resolves and passes beyond the Xmode cut-off (see Fig. 8). When the hole resolves before passing the cut-off, radiation will be in the $\mathrm{Z}$ mode.

bility to $1500<t \omega_{\mathrm{e}} \lesssim 3000$ (Newman et al., 2002). This corresponds to $t \omega_{\text {ce }} \gtrsim 3.6 \times 10^{5}$ for the FAST 1773 observations. Compared to the growth time obtained above this implies roughly $\sim 70$ e-foldings of the wave during complete trapping, or a huge - and thus unrealistically high - amplification factor in our case. We may thus realise that radiation trapping inside the hole might be a viable process for keeping the radiation in resonance and amplifying it over the lifetime of the hole until being released from the hole. But trapping cannot be complete because the amplification would become excessively large. In the downward current region lifetimes of the above given length correspond to times $0.5 \mathrm{~s}<t<1 \mathrm{~s}$ and to propagation distances of $5 \times 10^{3} \mathrm{~km}<L_{\mathrm{h}}<10^{4} \mathrm{~km}$ of the hole along the magnetic field at nominal hole velocity $V_{\mathrm{h}} \lesssim\langle V\rangle \sim 10^{4} \mathrm{~km} \mathrm{~s}^{-1}$. These distances are not only somewhat large in the auroral region, they yield, in addition, unreasonably high amplification factors of the order of $10^{13}$ or so. Roughly ten times shorter lifetimes would cause lower amplification rates but are in disagreement with simulations. Moreover, for trapping of wavelengths of the order of $1 \mathrm{~km}$ the perpendicular size of the holes should be of same order. This is suggested by the observations of about symmetrical holes with comparable parallel and perpendicular dimensions (Franz et al., 2000) but has theoretically not yet been confirmed. As mentioned before, the hole extension is up to few kilometers along the magnetic field. The perpendicular extension is not precisely known. It may range from $100 \mathrm{~m}$ 
to few $\mathrm{km}$, depending on the symmetry of the hole. Shorter perpendicular sizes cause severe problems because the wave frequency is fixed to $\omega<\omega_{\mathrm{ce}}$, not permitting for large perpendicular wavenumbers $k_{\perp} \sim \omega / c$ and much shorter than $\sim \mathrm{km}$ wavelengths. Waves at these frequencies are inhibited to propagate outside the hole where they become evanescent. It might be speculated that the cyclotron maser instability pumped energy into the wave only over the fraction of wavelength inside the hole and that most of this energy would be absorbed on the fraction outside the hole. However, the confirmation of this speculation requires an eikonal solution of the long wave amplification problem.

\subsection{Mechanisms reducing amplification}

On the other hand, accepting long lifetimes and assuming symmetric holes in configuration space, one requires that any excessive amplification must be reduced by some mechanism inside the hole during trapping time. Three such mechanisms can be envisaged: self-absorption of the radiation amplified and trapped inside the hole, quasi-linear saturation of trapped amplified radiation, and leakage of trapped amplified radiation from the hole during upward propagation of the hole to decreasing magnetic fields. All three mechanisms are indeed possible for holes in the downstream current region. We will discuss the two former and leave the question of radiation escape to the next section. In fact, untrapping might limit the amplification rate even more than both above restrictions.

(a) Self-absorption: Holes move up along the magnetic field from regions of strong magnetic fields into regions of low magnetic fields. We have shown (Treumann et al., 2011b) that the interior of electron holes is capable of absorption of wave energy at a weaker rate than emission. This is due to the hole being filled with a low density hot electron component the phase space density of which exhibits negative perpendicular velocity gradients $\partial f_{\text {trapped }} / \partial v_{\perp}<0$. The frequency of absorption is higher than the frequency of emission, $\omega_{\text {abs }}>\omega$.

Motion of the hole during its lifetime from higher to lower magnetic fields shifts the emission, amplification and absorption to ever lower frequencies (see Fig. 7). By this a wave which had been emitted at some frequency $\omega$ in the high $\omega_{\text {ce }}$ region shifts out of resonance and amplification entering into the absorption band where it becomes damped, though at a slower rate. A mechanism of this kind might limit the amplification of the radiation and reduce its intensity to observed values. It, at the same time, increases the bandwidth of radiation to cover the frequency gap between the final cyclotron and emission frequencies reached when the radiation is released from the hole. A sketch of this mechanism is given in Fig. 8.

One may get an impression of the downward resonant frequency shift assuming a geomagnetic dipole field $B=$ $B_{0} / R^{3}$ with $B_{0}$ the magnetic field at Earth's surface $r=R_{\mathrm{E}}$,

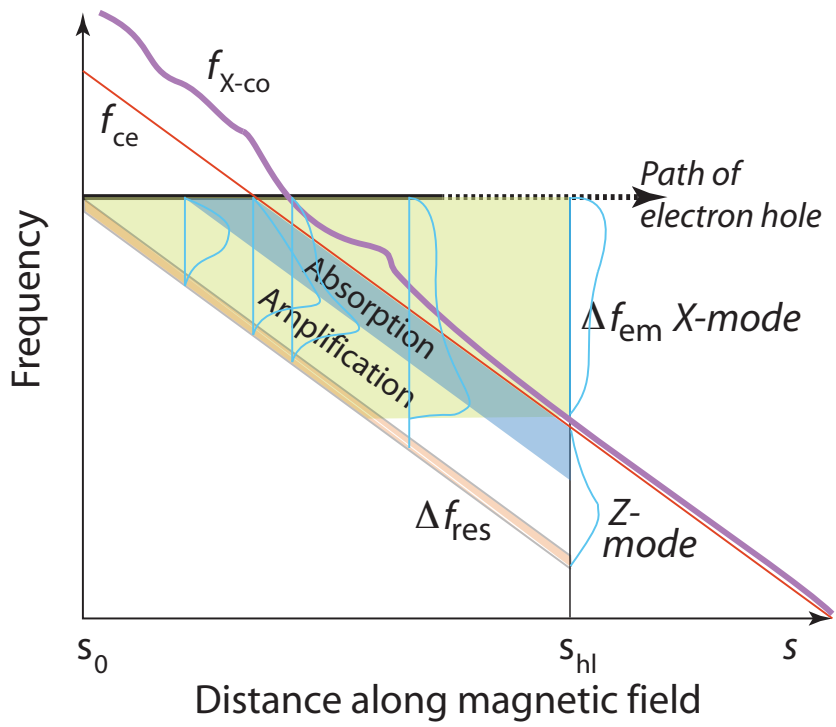

Fig. 8. The evolution of the emission spectrum during passage of the electron hole along the magnetic field from high to low magnetic field strengths (upward). The coordinate $s$ is distance along the magnetic field line, $s_{0}$ the start location of the hole. Schematically shown is the variation of the cyclotron frequency $f_{\text {ce }}=\omega_{\text {ce }} / \omega_{\text {ce } 0} \approx 1-3\left(s-s_{0}\right) /\left(R_{\mathrm{E}}+s_{0}\right)$ in a dipole field, and the $\mathrm{X}$-mode cut-off frequency. The horizontal line is the path of the hole until hole dissolution at location $s_{\mathrm{hl}}=v_{\mathrm{h}} \tau_{\mathrm{h}}$, with $\tau_{\mathrm{h}}$ the hole life time. The evolution of the emission $\Delta f_{\text {res }}$, amplification, and absorption ranges is indicated. A wave which was excited at some location $s \gtrsim s_{0}$ by the maser mechanism at a frequency in the narrow frequency range $\Delta f_{\text {res }}$ will necessarily pass into the amplification and absorption bands when the hole moves up the field line to lower fields. It will become free, if and after the hole passes the cut-off and subsequently dissolves. The vertical blue cuts show the expected spectra at 4 locations. These are modulated by emission, amplification and absorption. After the hole passed the cut-off frequency and resolves, the final emission is released in the $\mathrm{X}$ mode over the bandwidth $\Delta f_{\mathrm{em}}$. In case the hole resolves before passing the cut-off, the radiation will be in the Z-mode and will be trapped in the downward current region plasma save passing the boundary to the upward current region where it transforms into the $\mathrm{X}$-mode.

and $R=r / R_{\mathrm{E}}=(1+\Delta s)-$ neglecting any latitudinal variation over the short distance $\Delta s=V_{\mathrm{h}} \Delta t$ (measured in $R_{\mathrm{E}}$ ) a hole travels at speed $V_{\mathrm{h}}$ during lifetime $\Delta t$. The resonance radius $R_{\text {res }}=$ const is fixed for the given electron distribution. Thus, the resonance frequency varies as the magnetic field, i.e.

$\partial \omega_{\mathrm{res}} \approx-3 \omega_{\mathrm{ce}}\left(V_{\mathrm{h}} / R_{\mathrm{E}}\right) \partial t$

At speed $V_{\mathrm{h}}$ few times $10^{3} \mathrm{~km} \mathrm{~s}^{-1}$ this implies for the FAST 1773 passage a substantial decrease in resonant emission frequency of $\partial \omega_{\text {res }} \sim 5 \% \sim 20 \mathrm{kHz}$. A wave amplified during the first phase of trapping is quickly shifted in frequency out of resonance into the absorption range thus reducing the amplification by the required order of magnitude 
from $\sim 70$ e-foldings to $\lesssim 10$ e-foldings, i.e. to a reasonable amplification factor of $\sim 10^{4}$ in wave amplitude and $\sim 10^{8}$ in wave power.

A quantitative estimate of the amplification/absorption rate is hardly possible without gathering more knowledge about the real velocity of the hole, its extension, the trapped number and energy densities, and the location of the holes. Previously (Treumann et al., 2011a, based on their Fig. 2) determined the background noise (whether instrumental or natural remained unclear) of the radiation power spectral density (gauging the weakest radiation level with the help of the colour bar) to $2 \pi \mathcal{P}_{\mathrm{b}} / \Delta \omega=2 \pi E^{2} / c \mu_{0} \Delta \omega \sim(8.4 / \Delta \omega) \times$ $10^{-16} \mathrm{~W} \mathrm{~m}^{-2} \mathrm{~Hz}$. (The power flux $\mathcal{P}$ would be obtained multiplying with the bandwidth $\Delta \omega / 2 \pi$.)

The maximum radiated power spectral density in the fine structures was of order $2 \pi \mathcal{P}_{\omega} / \Delta \omega \sim(2.7 / \Delta \omega) \times$ $10^{-11} \mathrm{~W} \mathrm{~m}^{-2} \mathrm{~Hz}$, corresponding to an amplification factor of $\mathcal{P}_{\omega} / \mathcal{P}_{\mathrm{b}} \sim 3.2 \times 10^{4}$. These numbers are in reasonable agreement with the above estimate for the downward current region. One may thus adopt the philosophy of the present paper that much of the fine structure of the auroral kilometric radiation would rather be generated in the downward than in the upward current region.

(b) Quasilinear saturation: This is indeed another possibility ${ }^{1}$ to be envisaged for limiting the amplification of radiation during trapping inside the hole.

Trapping of radiation inside the hole keeps the radiation in resonance until displacement of the hole along the magnetic field shifts the wave out of resonance. If this time is long enough for the radiation to intensify sufficiently, quasilinear saturation will limit any further amplification and deplete the positive phase-space gradient at the hole boundary by plateau formation and heating the resonant electrons. This causes weakening and finally destruction of the hole.

A quantitative estimate of this mechanism cannot be given without developing the quasilinear theory of the electron cyclotron maser inside the hole which will not be attempted in the present communication. Nevertheless, in contrast to quasilinear saturation by trapping in the auroral cavity, where the radiation will barely encounter resonance many times again at its original emission frequency, the quasilinear saturation mechanism seems viable for electron hole-trapped radiation and, thus, possibly acts in combination with the former mechanism of self-absorption to limit unreasonably strong amplification.

\subsection{Release of radiation}

In the above scenario electron holes of appropriate size in the downward current region can indeed generate, amplify and self-absorb narrow-band radiation when moving up along the magnetic field from high to low magnetic field strengths at

\footnotetext{
${ }^{1}$ kindly suggested by the referees
}

velocity of few thousand kilometres per second for a limited lifetime.

Acknowledging this fact, one is confronted with the additional problem of how the radiation escapes from the holes and why it is observed throughout the entire auroral cavity as shown in Fig. 6. (We do not ask the more general question how it escapes from the auroral cavity into free space).

Hole-trapped and amplified radiation can escape from the hole once the hole decays and has moved up to regions of magnetic field strengths low enough for the trapped radiation to have frequency above the X-mode cut-off. The condition for this to happen is easy to check for the given ratio $\omega_{\mathrm{e}} / \omega_{\mathrm{ce}} \approx 1 / 20=0.05$ in the upward current region, yielding an X-mode cut-off frequency (e.g. Baumjohann and Treumann, 1996, pp. 228)

$\omega_{\mathrm{X}-\mathrm{co}} \approx \omega_{\mathrm{ce}}\left(1+\omega_{\mathrm{e}}^{2} / \omega_{\mathrm{ce}}^{2}\right) \approx 1.003 \omega_{\mathrm{ce}}$

which is only slightly higher than the upper-hybrid frequency. At $\omega_{\mathrm{ce}} / 2 \pi=480 \mathrm{kHz}$ the cut-off is just $\sim 1 \mathrm{kHz}$ above the electron cyclotron frequency. Hence, by the above given estimate of frequency shift, the hole needs only a fraction $\Delta t / 20$ of its lifetime for the frequency of the trapped radiation to exceed the external X-mode cut-off. It is therefore no problem for any radiation that is trapped in the hole to find itself in free space above the local X-mode cut-off after the upward moving hole has moved sufficiently high up on the field line. The radiation can then leak out and escape into the auroral cavity possibly even without complete destruction of the hole. In this case untrapping determines the limitation and ultimate intensity of the electron-hole maser radiation in the downward current region (as shown in Fig. 8). Being emitted perpendicular to the magnetic field in the downward current region, the radiation finds its way into the upward current region where densities and cut-offs are even lower. In agreement with observation it will be observed throughout the entire auroral cavity with no distinction being made between upward and downward currents.

\section{Conclusions}

The downward current region does not host electron velocity distributions with positive perpendicular velocity space gradients. Such gradients can, however, be caused on microscopic scales by electron holes, solitons or double layers. We argued (Treumann et al., 2011a) that they will be the rule whenever electron holes (or similar structures) evolve, in which case electron holes become electron-cyclotron emitters and absorbers. In the upward current region the theory faced some hardly solvable problems. However, in the downward current region the problems became less severe offering reasonable solutions. Growth rates for radiation obtained there were high compared to the upward current region.

Problems arose with amplification and trapping of radiation inside holes. Trapping is possible for holes of 
comparable parallel and perpendicular spatial sizes. In this case large amplification rates were obtained. However, this problem turned out to be less restrictive. Self-absorption of radiation during upward motion of the holes, quasilinear saturation and radiation leakage all keep the amplification low. Release of radiation after amplification from holes in the upward current region seems responsible for at least part of the observed auroral kilometric radiation fine structure. The theory has been developed in the hole frame. Transformation into the observer's frame takes care of the hole speed and causes Doppler shift in frequency and obliquity of radiation.

Thus, for the emission of auroral kilometric radiation by the electron-cyclotron maser mechanism, the downward current region turns out to be as important as the upward current region. While the upward current region, because of the presence of loss cone and ring-horseshoe distributions, may dominate the generation of the auroral kilometric background continuum, production of fine structure is probably added from the downward current region by processes of the kind described - or similar ones involving other microscopic structures like double layers, solitons or ion holes, all producing density gradients. The sole condition is that the structure is capable of generating a velocity space gradient on the electron distribution on the spatial scale of the structure. We stress, that the generation of fine structure in the upward current region is not excluded by our theory. It may happen in cases when sufficiently steep perpendicular velocity space gradients evolve on horseshoe or loss-cone distributions or radiation from upward current region electron holes becomes sufficiently amplified. The present theory provides an alternative which may be naturally realised.

We note finally that such a model might be applicable to other planets, the sun, magnetised stars, and also some astrophysical objects like non-relativistic and relativistic shocks (Treumann, 2009; Bykov and Treumann, 2011) as well where the identification of the emitted radiation as cyclotron-maser radiation would indicate the presence of strongly amplified magnetic fields and localised plasma depletion by intense magnetic field-aligned electric fields inside electron holes.

Acknowledgements. This research was part of an occasional Visiting Scientist Programme in 2006/2007 at ISSI, Bern. RT thankfully recognises the assistance of the ISSI librarians, Andrea Fischer and Irmela Schweizer. He appreciates the encouragement of André Balogh, Director at ISSI. The FAST spacecraft observations used have been obtained within the University of California at Berkeley - France cooperation. The data in Fig. 1 have previously been published. They are reprinted here (with changes) with the permission of the American Geophysical Union. The spectra in Figs. 1 and 2 are based on those data. Some of the data work was done more than ten years ago with the help and technical expertise of Edita Georgescu which we gratefully acknowledge at this place. RT also acknowledges the insisting sceptical comments of the anonymous referees of the former paper (Treumann et al., 2011a) on electron holes in the upward current region which led to the re-thinking of a possible contribution of the downward current region to auroral kilometric maser radiation. He also acknowledges the helpful remarks of the referees, in particular the suggestion that quasilinear saturation of trapped radiation inside holes might also limit amplification, a mechanism which still has to be investigated in detail. Regrettably, in spite of their positive inclinations, the referees preferred anonymity. As it is always at risk, proposing unconventional mechanisms of explanation of long-standing unsatisfactorily understood physical effects, we hope that their preference does not indicate any hidden mistrust.

Topical Editor I. A. Daglis thanks two anonymous referees for their help in evaluating this paper.

\section{References}

Andersson, L., Ergun, R. E., Newman, D. L., McFadden, J. P., and Carlson, C. W.: Characteristics of parallel electric fields in the downward current region of the aurora, Phys. Plasmas, 9, 3600, doi:10.1063/1.1490134, 2002.

Baumjohann, W. and Treumann, R. A.: Basic Space Plasma Physics, Imperial College Press, London, pp. 228-234, 1996.

Berman, R. H., Dupree, T. H., and Tetreault, D. J.: Growth of nonlinear intermittent fluctuations in linearly stable and unstable simulation plasma, Phys. Fluids, 29, 2860-2870, doi:10.1063/1.865486, 1986.

Buneman, O.: Instability, turbulence, and conductivity in current-carrying plasma, Phys. Rev. Lett., 1, 8-9, doi:10.1103/PhysRevLett.1.8, 1958.

Buneman, O.: Dissipation of currents in ionized media, Phys. Rev., 115, 503-517, doi:10.1103/PhysRev.115.503, 1959.

Bykov, A. M. and Treumann, R. A.: Fundamentals of collisionless shocks for astrophysical application, 2. Relativistic shocks, Astron. Astrophys. Rev., 19, 42, doi:10.1007/s00159-011-0042-8, arXiv:1105.3221, 2011.

Carlson, C. W., McFadden, J. P., Ergun, R. E., Temerin, M., Peria, W., Mozer, F. S., Klumpar, D. M., Shelley, E. G., Peterson, W. K., Moebius, E., Elphic, R., Strangeway, R., Cattell, C., and Pfaff, R.: FAST observations in the downward auroral current region: Energetic upgoing electron beams, parallel potential drops, and ion heating, Geophys. Res. Lett., 25, 2017-2020, doi:10.1029/98GL00851, 1998.

Ergun, R. E., Carlson, C. W., McFadden, J. P., Mozer, F. S., Delory, G. T., Peria, W., Chaston, C. C., Temerin, M., Elphic, R., Strangeway, R., Pfaff, R., Cattell, C. A., Klumpar, D., Shelley, E., Peterson, W., Moebius, E., and Kistler, L.: FAST satellite observations of electric field structures in the auroral zone, Geophys. Res. Lett., 25, 2025-1028, doi:10.1029/98GL00635, 1998a.

Ergun, R. E., Carlson, C. W., McFadden, J. P., Mozer, F. S., Delory, G. T., Peria, W., Chaston, C. C., Temerin, M., Roth, I., Muschietti, L., Elphic, R., Strangeway, R., Pfaff, R., Cattell, C. A., Klumpar, D., Shelley, E., Peterson, W., Moebius, E., and Kistler, L.: FAST satellite observations of largeamplitude solitary structures, Geophys. Res. Lett., 25, 20412044, doi:10.1029/98GL00636, 1998b.

Ergun, R. E., Carlson, C. W., McFadden, J. P., Mozer, F. S., Muschietti, L., Roth, I., and Strangeway, R. J.: Debye-scale plasma structures associated with magnetic-field-aligned electric fields, Phys. Rev. Lett., 81, 826-829, doi:10.1103/PhysRevLett.81.826, 1998c. 
Ergun, R. E., Andersson, L., Main, D., Su, Y.-J., Carlson, C. W., McFadden, J. P., and Mozer, F. S.: Parallel electric fields in the upward current region of the aurora: Indirect and direct observations, Phys. Plasmas, 9, 3685-3694, doi:10.1063/1.1499120, 2002a.

Ergun, R. E., Andersson, L., Main, D., Su, Y.-J., Newman, D. L., Goldman, M. V., Carlson, C. W., McFadden, J. P., and Mozer, F. S.: Parallel electric fields in the upward current region of the aurora: Numerical solutions, Phys. Plasmas, 9, 3695-3704, doi:10.1063/1.1499121, 2002b.

Franz J. R., Kintner, P. M., Seyler, C. E., Pickett, J. S., and Scudder, J. D.: On the perpendicular scale of electron phase-space holes, Geophys. Res. Lett., 27, 169-1672, doi:10.1029/1999GL010733, 2000.

Gray, P. C., Hudson, M. K., Bergmann, R., and Roth, I.: Simulation study of ion two-stream instability in the auroral acceleration region, Geophys. Res. Lett., 17, 1609-1612, doi:10.1029/GL017i010p01609, 1990.

Hess, S., Mottez, F., and Zarka, P.: Effect of electric potential structures on Jovian S-burst morphology, Geophys. Res. Lett., 36, 14101, doi:10.1029/2009GL039084, 2009.

Louarn, P.: Generation of auroral kilometric radiation in bounded source regions, in: Geospace Electromagnetic Waves and Radiation, Lecture Notes in Physics (LNP) Vol. 687, Springer-Verlag, Berlin-Heidelberg-New York, pp. 55-86, 2006.

Louarn, P., Kurth, W. S., Gurnett, D. A., Hospodarsky, G. B., Persoon, A. M., Cecconi, B., Lecacheux, A., Zarka, P., Canu, P., Roux, A., Rucker, H. O., Farrell, W. M., Kaiser, M. L., Andre, N., Harvey, C., and Blanc, M.: Observation of similar radio signatures at Saturn and Jupiter: Implications for the magnetospheric dynamics, Geophys. Res. Lett., 34, L20113, doi:10.1029/2007GL030368, 2007.

Mottez, F., Hess, S., and Zarka, P.: Explanation of dominant oblique radio emission at Jupiter and comparison to the terrestrial case, Planet. Space Sci., 58, 1414-1422, doi:10.1016/j.pss.2010.05.012, 2010.

Muschietti, L., Ergun, R. E., Roth, I., and Carlson, C. W.: Phasespace electron holes along magnetic field lines, Geophys. Res. Lett., 26, 1093-1096, doi:10.1029/1999GL900207, 1999a.

Muschietti, L., Roth, I., Ergun, R. E., and Carlson, C. W.: Analysis and simulation of BGK electron holes, Nonlin. Processes Geophys., 6, 211-219, doi:10.5194/npg-6-211-1999, 1999 b.

Newman, D. L., Goldman, M. V., Ergun, R. E., and Mangeney, A.: Formation of Double Layers and Electron Holes in a Current-Driven Space Plasma, Phys. Rev. Lett., 87, 255001, doi:10.1103/PhysRevLett.87.255001, 2001.

Newman, D. L., Goldman, M. V., and Ergun, R. E.: Evidence for correlated double layers, bipolar structures, and very-lowfrequency saucer generation in the auroral ionosphere, Phys. Plasmas, 9, 2337-2343, doi:10.1063/1.1455004, 2002.

Oppenheim, M. M., Vetoulis, G., Newman, D. L., and Goldman, M. V.: Evolution of electron phase-space holes in 3D, Geophys. Res. Lett., 28, 1891-1894, doi:10.1029/2000GL012383, 2001.

Pottelette, R. and Pickett, J.: Phase space holes and elementary radiation events, Nonlin. Processes Geophys., 14, 735-742, doi:10.5194/npg-14-735-2007, 2007.
Pottelette, R. and Treumann, R. A.: Electron holes in the auroral upward current region, Geophys. Res. Lett., 1432, L12104, doi:10.1029/2005GL022547, 2005.

Pottelette, R., Treumann, R. A., and Berthomier, M.: Auroral plasma turbulence and the cause of auroral kilometric radiation fine structure, J. Geophys. Res., 106, 8465-8476, doi:10.1029/2000JA000098, 2001.

Pritchett, P. L.: Relativistic dispersion and the generation of auroral kilometric radiation, Geophys. Res. Lett., 11, 143-146, doi:10.1029/GL011i002p00143, 1984a.

Pritchett, P. L.: Relativistic dispersion, the cyclotron maser instability, and auroral kilometric radiation, J. Geophys. Res., 89, 89578970, doi:10.1029/JA089iA10p08957, 1984b.

Pritchett, P. L.: Electron cyclotron maser radiation from a relativistic loss-cone distribution, Phys. Fluids, 27, 2393-2396, doi:10.1063/1.864542, 1984c.

Pritchett, P. L.: Electron cyclotron maser instability in relativistic plasmas, Phys. Fluids, 29, 229-315, doi:10.1063/1.865492, 1986.

Tetreault, D. J.: Growing ion holes as the cause of auroral double layers, Geophys. Res. Lett., 15, 164-167, doi:10.1029/GL015i002p00164, 1988.

Tetreault, D.: Theory of electric fields in the auroral acceleration region, J. Geophys. Res., 96, 3549-3563, doi:10.1029/90JA02324, 1991.

Treumann, R. A.: The electron-cyclotron maser for astrophysical application, Astron. Astrophys. Rev., 13, 229-315, doi:10.1007/s00159-006-0001-y, 2006.

Treumann, R. A.: Fundamentals of collisionless shocks for astrophysical application, 1. Non-relativistic shocks, Astron. Astrophys. Rev., 17, 409-535, doi:10.1007/s00159-009-0024-2, 2009.

Treumann, R. A. and Baumjohann, W.: Advanced Space Plasma Physics, Imperial College Press, London, pp. 21-25, 1997.

Treumann, R. A., Jaroschek, C. H., and Pottelette, R.: On deformation of electron holes in phase space, Europhys. Lett., 84, 69001, doi:10.1209/0295-5075/84/69001, 2008.

Treumann, R. A., Treumann, R. A., Baumjohann, W., and Pottelette, R.: Electron-cylotron maser radiation from electron holes: upward current region, Ann. Geophys., 29, 1885-1904, doi:10.5194/angeo-29-1885-2011, 2011a.

Treumann, R. A., Nakamura, R., and Baumjohann, W.: A model of so-called "Zebra" emissions in solar flare radio burst continua, Ann. Geophys., 29, 1673-1682, doi:10.5194/angeo-291673-2011, $2011 b$.

Winglee, R. M.: Interrelation between azimuthal bunching and semirelativistic maser cyclotron instabilities, Plasma Phys., 25, 217-255, doi:10.1088/0032-1028/25/2/006, 1983.

Wu, C. S. and Lee, L. C.: A theory of the terrestrial kilometric radiation, Astrophys. J., 230, 621-626, doi:10.1086/157120, 1979.

Zarka, P. and Kurth, W. S.: Radio wave emission from the outer planets before Cassini, Space Sci. Rev., 116, 371-397, doi:10.1007/s11214-005-1962-2, 2005. 